\title{
BMJ Open Study protocol for a multi-institutional, randomised, double-blinded, placebo- controlled phase III trial investigating additive efficacy of duloxetine for neuropathic cancer pain refractory to opioids and gabapentinoids: the DIRECT study
}

\author{
Hiromichi Matsuoka, ${ }^{1,2}$ Hiroto Ishiki, ${ }^{3}$ Satoru Iwase, ${ }^{4}$ Atsuko Koyama, ${ }^{1,2}$ \\ Takashi Kawaguchi, ${ }^{5}$ Yoshiyuki Kizawa, ${ }^{6}$ Tatsuya Morita, ${ }^{7}$ Yoshinobu Matsuda, ${ }^{8}$ \\ Tempei Miyaji, ${ }^{9}$ Keisuke Ariyoshi, ${ }^{4,10}$ Takuhiro Yamaguchi ${ }^{9,11}$
}

To cite: Matsuoka $\mathrm{H}$, Ishiki $\mathrm{H}$, Iwase S, et al. Study protocol for a multi-institutional, randomised, double-blinded, placebo-controlled phase III trial investigating additive efficacy of duloxetine for neuropathic cancer pain refractory to opioids and gabapentinoids: the DIRECT study. BMJ Open 2017;7:e017280. doi:10.1136/ bmjopen-2017-017280

- Prepublication history for this paper is available online. To view these files please visit the journal online (http://dx.doi org/10.1136/bmjopen-2017017280).

Received 30 April 2017 Revised 6 July 2017 Accepted 6 July 2017

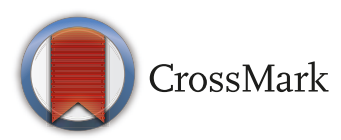

For numbered affiliations see end of article.

Correspondence to Dr Hiromichi Matsuoka; matsuoka_h@med.kindai.ac.jp

\section{ABSTRACT}

Introduction Management of patients with cancer suffering from neuropathic pain refractory to opioids and gabapentinoids remains an important challenge. Duloxetine is one of the choices after first-line treatment fails. The efficacy of duloxetine has been reported in patients with non-cancer disease and in chemotherapyinduced peripheral neuropathy, but no randomised clinical trials have examined its effects on neuropathic cancer pain refractory to first-line treatment. The objective of this study is to assess the analgesic efficacy of duloxetine in patients suffering from neuropathic cancer pain refractory to opioids and gabapentinoids.

Methods and analysis A multi-institutional, prospective, randomised, double-blind, placebo-controlled, two-parallel trial is planned. The inclusion criteria are adult patients with cancer suffering from neuropathic cancer pain refractory to opioids and gabapentinoids, patients with a Numerical Rating Scale (NRS) pain score of 4 or higher and patients with a total Hospital Anxiety and Depression Scale score of less than 20. Patients with chemotherapyinduced peripheral neuropathy are excluded. The study will take place at 14 sites across Japan. Participants will be randomised (1:1 allocation ratio) to a duloxetine intervention group or a placebo control group. Evaluations will be made at baseline (T0 randomisation), day 0 (T1), day 3 (T2) and day 10 (T3). The primary endpoint is defined as the difference in NRS score for pain intensity (average over the previous 24 hours) at T3 between the duloxetine and placebo groups. A sample size of 70 patients will be examined between July 2015 and March 2018.

Ethics and dissemination Ethics approval was obtained at all participating sites. The results of this study will be submitted for publication in international peer-reviewed journals and the key findings presented at international scientific conferences.

Trial registration number UMIN000017647; Pre-results. Protocol version 2.2, 26 April 2017.
Strengths and limitations of this study

This is the first study to assess the analgesic efficacy of duloxetine in patients suffering from neuropathic cancer pain refractory to opioids and gabapentinoids, and the results of the trial will clarify the second-line standard treatment for cancerrelated neuropathic cancer pain.

- This is an adequately powered study to provide a clinically meaningful outcome, and adverse effects following interventions will be systematically evaluated.

- We excluded patients with depression from the study population for accurate evaluation of the pharmacological effects of duloxetine on pain.

- This study includes the heterogeneity of causes of neuropathic cancer pain.

- The primary endpoint is not worst pain intensity in the last 24 hours but the difference in average pain intensity score at T3 (day 10) between two groups.

\section{INTRODUCTION}

Pain is a symptom that is experienced by many patients with cancer. Prevalence at the time of diagnosis is $30 \%$, and this increases to $90 \%$ in advanced stages. ${ }^{12}$ Pain in patients with cancer is often classified as nociceptive pain or neuropathic pain (NP), but both types are thought to be intermixed. Of patients with cancer-related pain, $33 \%$ have NP. ${ }^{1}$ In general, NP is often resistant to treatment, whereas nociceptive pain tends to respond well to treatment. ${ }^{2-5}$

The efficacy of many drugs for NP has been reported in patients with non-cancer 
disease, and some have been shown to be effective for NP in patients with cancer. ${ }^{6}$ These drugs include opioids, tricyclic antidepressants (TCAs) and gabapentinoids (gabapentin and pregabalin).$^{7-11}$

In patients with cancer, the efficacy of TCAs and gabapentinoids has been proven in clinical trials, ${ }^{12}{ }^{13}$ and a phase III study revealed moderate analgesic effects of gabapentin in combination with opioids. ${ }^{14}$ However, it is unclear which drug is most effective in cases in which first-line treatment with gabapentinoids fails to alleviate $\mathrm{NP}$ in patients with cancer.

In treatment of non-cancer NP, the efficacy of addition of duloxetine to pregabalin has been shown in a phase III study. ${ }^{15}$ There is, however, no empirical data for second-line treatment of NP in patients with cancer. The National Comprehensive Cancer Network guidelines list duloxetine as a potential choice for second-line treatment, ${ }^{8}$ and a phase III study showed the efficacy of duloxetine in treatment of chemotherapy-induced peripheral neuropathy (CIPN) in patients with cancer. ${ }^{16}$ Furthermore, a small retrospective study reported the beneficial effects of duloxetine in palliation of NP refractory to pregabalin and opioids in 15 patients with cancer. ${ }^{17}$ On the other hand, duloxetine has the potential for adverse events (AEs) such as dry mouth, sweating, fatigue, nausea, constipation, loss of appetite, dizziness, diarrhoea, hot flashes, hypertension, hyperhidrosis, palpitations, insomnia and drug-drug interactions as well as a risk of serotonergic syndrome and therefore should be used carefully. ${ }^{18-20}$ According to a meta-analysis in 2015, ${ }^{12}$ seven studies demonstrated clinical effects of duloxetine, but two studies revealed negative results.

In the double-blind, randomised, placebo-controlled study described here, we will evaluate the efficacy of addition of duloxetine for neuropathic cancer pain refractory to opioids and gabapentinoids. Currently, there is no specific standard treatment for NP in patients with cancer; placebo is used instead of active control. The results of the trial will clarify the second-line standard treatment for cancer-related NP.

\section{METHODS AND ANALYSIS \\ Study design}

The SPIRIT (Standard Protocol Items for Randomised Trials) statement and its checklist were followed in preparing the protocol. The study design is summarised in figure 1. A multicentre, prospective, randomised, placebo-controlled, double-blinded, two-parallel group study will be performed to compare the efficacy of addition of duloxetine (intervention group) with the efficacy of addition of a placebo (control group).

\section{Study settings and participants}

Recruiting will be performed in 14 adult palliative care sites across Japan, with involvement of 10 palliative care teams and 4 palliative care units. The inclusion and exclusion criteria are summarised in box 1 .
The main inclusion criterion is patients suffering from cancer pain (neuropathic or mixed) refractory to opioids and gabapentinoids. Diagnosis of NP is based on the International Association for the Study of Pain algorithm, in which a diagnosis of NP is made for patients with (1) pain with a distinct neuroanatomically plausible distribution, (2) a history suggestive of a relevant lesion or disease affecting the peripheral or central somatosensory system, (3) a range of pain that is neuroanatomically plausible and symptoms suggesting somatosensory injury or neurological disease (ie, hyperalgesia, hypoalgesia, dysesthesia or allodynia along the dermatome) and (4) relevant objective or imaging findings suggesting nervous system injury or disease (ie, imaging findings showing that a lesion is present). Based on these criteria, the certainty of the presence of NP is graded as definite NP ( 1 to 4 present) and probable NP ( 1 and 2 , plus 3 or 4). ${ }^{21}$ Definite and probable NP will be considered to indicate NP, and patients with these conditions will be eligible as subjects. Patients with an average Numerical Rating Scale (NRS) pain score in the preceding 24-hour period of 4 or higher ${ }^{22}$ and those with total Hospital Anxiety and Depression Scale (HADS) scores of less than 20 will be included, based on criteria for pain intensity used in similar studies on NP. ${ }^{23}$ The exclusion criteria are patients with progressive paralysis, a known contraindication to use of duloxetine, or depression. To examine the effects of duloxetine, we believe it is necessary to exclude patients with depression because duloxetine may alleviate pain through improving depression. Patients with CIPN or impaired cognitive function will also be excluded.

\section{Recruitment, randomisation, masking and follow-up Recruitment}

Eligible patients satisfying the screening inclusion and exclusion criteria will be invited to participate in the study by site investigators.

\section{Randomisation}

Physicians will introduce the trial to patients. On enrolment and after providing informed consent, patients will be randomly allocated to intervention (duloxetine) or control (placebo) groups in a web-based central randomisation system using minimisation methods and a computer-generated randomisation schedule with a 1:1 allocation ratio. In performing this allocation, we will minimise the following adjustment factors to avoid a large bias: (1) average pain intensity measured by the NRS in the last 24 hours $(\leq 7, \geq 8)$, (2) type of pain (spinal cord infiltration or others), (3) HADS total score $(\leq 10, \geq 11)$, (4) treatment setting (inpatient or outpatient), (5) response to gabapentinoids (non-responsive or intolerance due to side effects) and (6) study site.

\section{Masking}

Patients and clinicians responsible for treatment will be blinded to administration of duloxetine or placebo. Only a clinical trial pharmacist who generate capsules, but is 


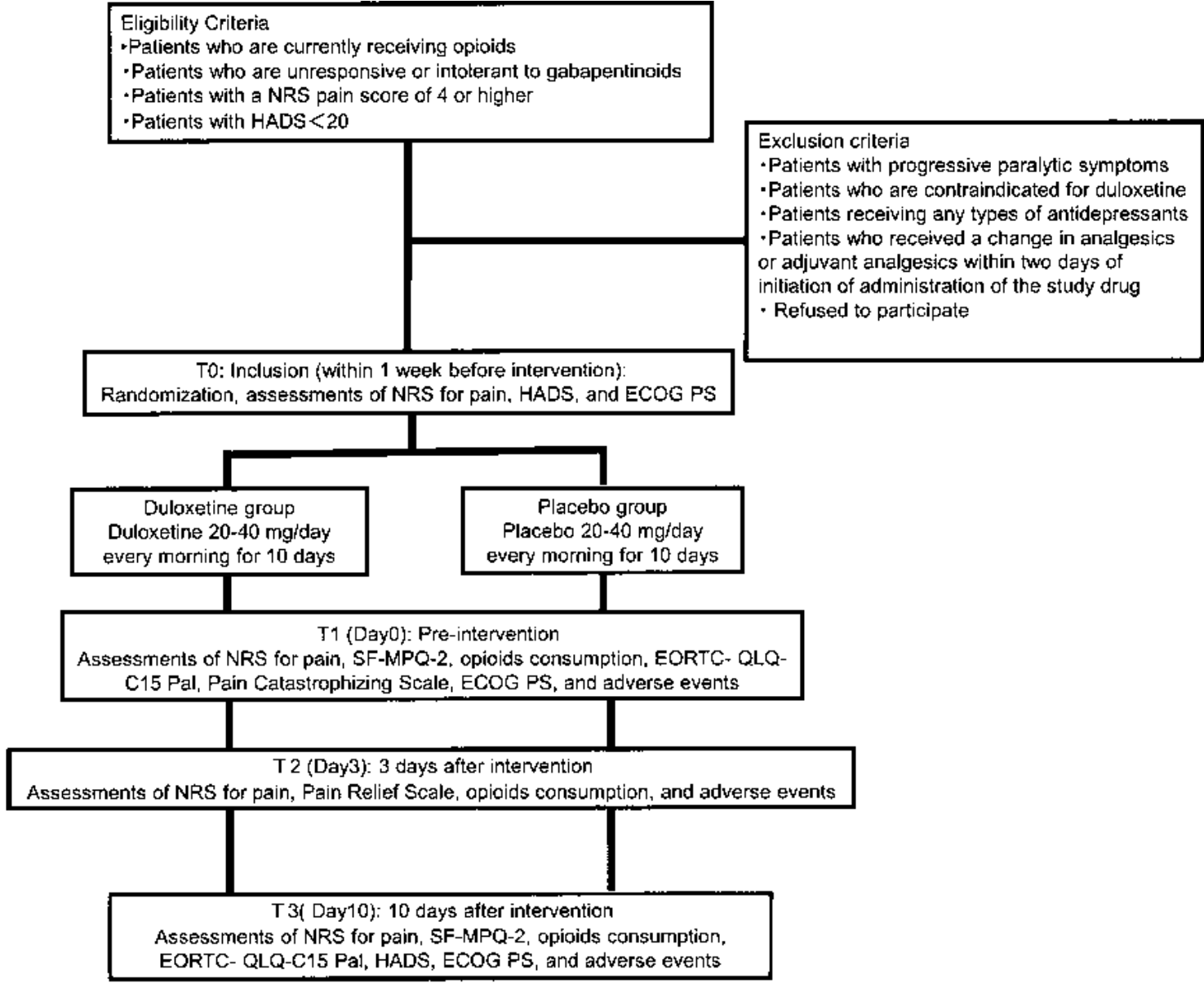

Figure 1 Flow chart of the procedures in the study. Participants will be randomised (1:1 allocation ratio) into the duloxetine intervention group or the placebo control group. Evaluations will be made at baseline (T0 randomization) and on day 0 (T1), day 3 (T2) and day 10 (T3). ECOG PS, European Cooperative Oncology Group Performance Status; EORTC, European Organisation for Research and Treatment of Cancer; HADS, Hospital Anxiety and Depression Scale; NRS, Numerical Rating Scale; SFMPQ-2, Short-Form McGill Pain Questionnaire 2.

not involved in patient care, will know the allocation and outcomes. All study drugs will be packaged by this pharmacist. Duloxetine (Cymbalta) will be administered with a change in dosage form: the capsules will be covered with a No. 3 capsule of the same material to make an overcapsule.

\section{Data management, central monitoring and audit}

Evaluations will be performed at four time points: baseline (time of randomisation, $\mathrm{T} 0$ ), the day before the start of treatment (day 0, T1) and 3 days (day 3, T2) and 10 days (day 10, T3) after initiation of treatment. The timing and details of evaluations are given in table 1 .

Once a patient is enrolled or randomised, the study site will make every reasonable effort to follow the patient for the entire study period. Patients will not be allowed to cross over from one group to another group until the end of the study (T3); however, they can choose to leave the study for any reasons at any time without detriment to the provision or quality of their clinical care. The investigators at each study sites will maintain individual records for each patient as source data, which include a copy of informed consent, medical records, laboratory data and other records or notes. All data will be collected by the independent data management centre. The data management centre will oversee the intrastudy data sharing process. The clinical data entry, data management and central monitoring will be performed using the electric data capture VIEDOC 3 (PCG Solutions, Sweden). An interim analysis will not be performed. Also, auditing is not planned in this study.

\section{Harms}

Investigators must record all AEs in the medical records and web systems. The National Cancer Institute's Common Terminology Criteria for Adverse Events (CTCAE) (V.4.0) will be used to grade each AE. All AEs are to be followed up continually during their course up. All severe adverse events must be reported to the institutional review board (IRB) and investigators in all sites and discussed through a FAX. Patients who are enrolled into the study will be treated by healthcare services provided by health insurance. 


\section{Box 1 Inclusion and exclusion criteria}

\section{Inclusion criteria}

- Inpatients and outpatients with diagnoses of cancer and neuropathic pain

- Currently receiving opioids

- Unresponsive or intolerant to gabapentinoids: (a) receiving doses of pregabalin of $300 \mathrm{mg} /$ day or higher or gabapentin of $1800 \mathrm{mg} /$ day or higher; (b) cannot receive increased doses of pregabalin or gabapentin due to side effects

- Numerical Rating Scale pain score of 4 or higher

- Hospital Anxiety and Depression Scale score $<20$

- Age 20 years or older

- Creatinine clearance rate $\geq 30 \mathrm{~mL} / \mathrm{min}$ (Cockcroft-Gault formula)

- Serum aspartate aminotransferase $<100 \mathrm{IU} / \mathrm{L}$, alanine aminotransferase $<100 \mathrm{IU} / \mathrm{L}$ and total bilirubin $<2.0 \mathrm{mg} / \mathrm{dL}$

$\checkmark$ Expected survival of 1 month or longer.

\section{Exclusion criteria}

- Chemotherapy-induced peripheral neuropathy

- Progressive paralytic symptoms

- Contraindication for duloxetine

- Taking any type of antidepressants

- A change in steroids, opioids, antidepressants, anticonvulsants, antipsychotic drugs, antiarrhythmic agents, or N-methyl-D-aspartate receptor antagonists within 2 days of initiation of administration of the study drug. Cases in which the patient has taken a hypnotic (including benzodiazepines such as zolpidem, zopiclone, eszopiclone, triazolam, ramelteon, suvorexant, brotizolam, flunitrazepam, rilmazafone and etizolam) as needed are not included.

- Drug misusers or patients who are addicted to drugs or have a history of addiction

- Patients with psychiatric disorders such as cognitive impairment who are unable to communicate

- Patients who are pregnant, breastfeeding or may possibly be pregnant

- Other patients who are determined to be inappropriate for participation in the study by the clinical investigator.

\section{Measurement tools}

Numerical Rating Scale

The 11-point NRS will be used to measure pain intensity from 0 (no pain) to 10 (worst possible pain), based on average pain in the past 24 hours. ${ }^{24}$

\section{Pain Relief Scale}

A self-assessment will be performed by the patients using the Pain Relief Scale. Patients will determine for themselves the efficacy of analgesics using a 4-point scale of complete relief, a lot of relief, slight relief and no change.

\section{Short-Form McGill Pain Questionnaire 2 (Japanese version)}

The Short-Form McGill Pain Questionnaire 2 (SF-MPQ-2) will be used to examine differences in effects due to pain mechanisms. The reliability and validity of the Japanese version have been verified. ${ }^{25}$

\section{European Organisation for Research and Treatment of Cancer QLQ-} C15-PAL

European Organisation for Research and Treatment of Cancer (EORTC) QLQ-C15-PAL will be used for evaluation of patient quality of life. The reliability and validity of the Japanese version have been confirmed. ${ }^{26}$

\section{Hospital Anxiety and Depression Scale}

The Hospital Anxiety and Depression Scale (HADS) will be used for measurement of psychiatric symptoms (anxiety and depression) of patients with a physical disease. HADS is a screening tool that allows assessment based on a small number of items. Its reliability and validity have been verified internationally. ${ }^{27} 28$ A HADS score $<20$ points as a cut-off for exclusion of patients with cancer with severe depression will be used, based on a previous report. $^{28}$

Table 1 Study procedure and time points for actions and evaluations

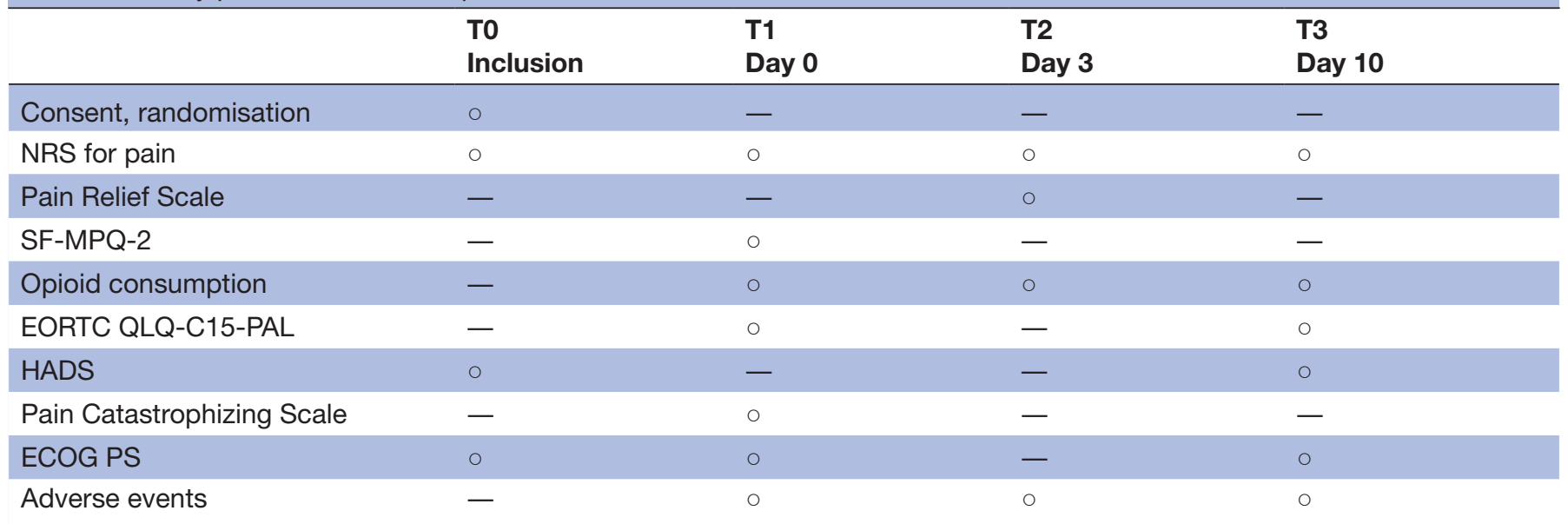

ECOG PS, European Cooperative Oncology Group Performance Status; EORTC, European Organisation for Research and Treatment of Cancer; HADS, Hospital Anxiety and Depression Scale; NRS, Numerical Rating Scale; SF-MPQ-2, Short-Form McGill Pain Questionnaire 2. 


\section{Pain Catastrophizing Scale}

The severity of cancer-related pain is influenced by engagement of patients in catastrophic thinking, such as 'my pain will undoubtedly never improve'. ${ }^{29}$ This effect will be measured using the Japanese version of the Pain Catastrophizing Scale (PCS), for which the validity and reliability have been shown. ${ }^{30}$

\section{Common Terminology Criteria for Adverse Events}

The worst grade of an AE during the preceding period will be assessed using the CTCAE V.4.0, Japanese Clinical Oncology Group (JCOG) version. Five AEs of somnolence, dizziness, nausea, palpitations and hypertension will be investigated if they occur at a relatively high frequency. Other AEs may also be assessed.

\section{Performance Status}

The European Cooperative Oncology Group Performance Status (ECOG PS) system will be used for evaluation of PS by primary physicians. ${ }^{31}$

\section{Treatment}

Preintervention treatment

Opioids (morphine, oxycodone, fentanyl, tapentadol) will be given to all patients. If AEs of gabapentinoids are severe, they will be discontinued or reduced in dose, but if the adverse effects are tolerable but the therapeutic effect is insufficient, gabapentinoids will be administered concomitantly with duloxetine. Pregabalin and gabapentin will be used in the study at the established effective doses of $300 \mathrm{mg}$ and $1800 \mathrm{mg}$, respectively. ${ }^{14} 32$

\section{Interventions}

Duloxetine or placebo will be administered for 10 days. Duloxetine $(20 \mathrm{mg} /$ day, one capsule) will be taken orally by participants in the intervention group starting after breakfast on day 1 . To determine if titration is necessary, pain relief will be evaluated using the Pain Relief Scale on day 3 (T2). Changes in symptoms, AEs and medication compliance will be evaluated. Patients who have 'complete relief' or 'a lot of relief' of pain will continue to receive doses of $20 \mathrm{mg}$ /day from day 4 . In all other cases, the dose will be increased to $40 \mathrm{mg} /$ day (two capsules) from day 4. If an intolerable $\mathrm{AE}$ such as nausea occurs at $40 \mathrm{mg} /$ day, the dose will be reduced as required. If an intolerable $\mathrm{AE}$ occurs at $20 \mathrm{mg} /$ day, the protocol will be discontinued. AEs that may be caused by duloxetine, such as somnolence, dizziness, nausea, palpitations and hypertension, will be evaluated to determine whether duloxetine treatment should be interrupted.

Placebo (22.4 mg lactose in a No. 4 capsule) will be administered to participants in the control group by oral administration (1 capsule/day) starting after breakfast on day 1 . To determine if titration is necessary, pain relief will be evaluated using the Pain Relief Scale on day 3 (T2), and changes in symptoms, AEs and medication compliance will be examined. Patients with 'complete relief' or 'a lot of relief' of pain will continue to receive 1 capsule/ day from day 4 . In all other cases, the dose will be increased to 2 capsules/day from day 4 . If an intolerable $\mathrm{AE}$ such as nausea occurs at 2 capsules/day, the dose will be reduced as side effects dictate. If there is an intolerable $\mathrm{AE}$ at 1 capsule/day, the protocol will be discontinued. To improve adherence to intervention protocols, participants will return the unused tablets at the last visit, and unused tablets will be counted and recorded on the medical records. Currently, there is no specific standard treatment for NP in patients with cancer; placebo is used instead of active control.

\section{Cotreatments}

Concomitantly administered analgesics such as opioids, non-steroidal anti-inflammatory drugs, acetaminophen or other adjuvant analgesics such as anticonvulsants, antidepressants, antiarrhythmics, N-methyl-D-aspartate receptor antagonists and steroids will not be changed during the follow-up period. In principle, new analgesics will not be started. When nausea occurs during the period of duloxetine administration, use of an antiemetic will be permitted. Currently used immediate-release opioids will be used in cases of breakthrough pain. Immediate-release rescue opioids will be freely permitted without limitation on the number of doses.

\section{Study endpoints}

Primary endpoint

The primary endpoint is a comparison of pain intensity (average pain over the previous 24hours) at T3 (Day 10) measured using NRS in the duloxetine and placebo groups.

\section{Secondary endpoints}

Efficacy will also be assessed using seven secondary endpoints: the nature of pain using SF-MPQ-2, EORTC QLQ-C15-PAL scores, daily opioid dose, changes in HADS score, degree of catastrophic thinking on PCS, AEs (CTCAE V.4.0-JCOG) and the difference in NRS scores for pain intensity (average over the previous 24hours) measured at T3 in subgroups of patients who are unresponsive or intolerant to gabapentinoids. Subgroup analyses will be performed on two patient groups; patients unresponsive to gabapentinoids and patients intolerant to gabapentinoids. Additionally, we will calculate percentages of the patients with $33 \%$ or $50 \%$ decrease.

\section{Statistical considerations}

\section{Statistical hypothesis}

Comparison of the primary endpoint of the NRS score for pain at day 10 (T3) (average pain over the previous 24-hour period) between the duloxetine and placebo groups will be conducted using a one-sided Student's t-test at a significance level of $5 \%$ according to the intentionto-treat principle. Point estimates and $90 \%$ CIs for the difference between two group means will be calculated.

The secondary endpoints of efficacy (SF-MPQ-2, EORTC QLQ-C15-PAL, HADS, PCS, daily opioid dose and group comparison of average pain on the NRS in the previous 24 hours in patients who are unresponsive 
or intolerant to gabapentinoids) will be evaluated similarly to the primary endpoint. The distribution of grades of AEs (CTCAE V.4.0-JCOG) and the incidence of AEs of grade 3 or higher and of grade 4 or higher will be determined. A Mantel test will be performed for group comparison.

\section{Sample size calculation}

The difference between group mean NRS scores for pain in the previous 24 hours on day 10 (T3) is assumed to be 1 point, and the SD of the NRS is taken to be 1.5 points. ${ }^{17}$ As there was no consensus about the minimal clinically important differences of duloxetine in cancer $\mathrm{NP}$ at the planning stage of the study, we decided to adopt a 1-point difference compared with placebo as the clinical significant difference, according to the meta-analysis of neuropathic non-cancer pain. ${ }^{33}$ During this study periods, Hui et al reported that the optimal cut-off was $\geq 1$ point for improvement in cancer pain. ${ }^{34}$

Assuming a rate of exclusion of $10 \%, 35$ subjects are needed in each group (70 subjects in total) to achieve a statistical power of $80 \%$ with a one-sided significance level of $5 \%$. As our primary interest is to clarify whether duloxetine is more effective than placebo, we adopted a one-sided test.

\section{Ethical issues}

All patients will be required to provide written informed consent. The study will be performed in accordance with the Declaration of Helsinki and the Japanese ethical guidelines for clinical research. The protocol was approved by the IRB at each study site (Osaka: Kindai University Hospital, Kansai Medical University Hospital, National Hospital Organization Kinki-Chuo Chest Medical Center, Sakai City Medical Center, Izumi Municipal Hospital and Sakai Hospital Kindai University Faculty of Medicine; Tokyo: National Cancer Center Hospital; Chiba: National Cancer Centre Hospital East; Nara: Nara Medical University Hospital; Nagoya: Nagoya University Hospital; Hyogo: Hyogo Prefectural Amagasaki General Medical Center, Kobe University Graduate School of Medicine and Hyogo Prefectural Kakogawa Medical Center; and Fukuoka: National Hospital Organization Kyusyu Cancer Center). This trial has been registered at the UMIN Clinical Trials Registry as UMIN000017647. Modifications in the study protocol will be communicated to the IRB at each study site as well as the independent data monitoring committee. Each ethics committee or IRB will revise informed consent materials given to participants and adapt according to their own institution's guidelines.

\section{DISCUSSION}

To our knowledge, there has been no randomised study of the analgesic efficacy of duloxetine in patients with neuropathic cancer pain refractory to opioids and gabapentinoids. In our planned trial, we will use a randomised, double-blind, placebo-controlled design, which is the most appropriate design to demonstrate the efficacy of a new therapy. Our findings using this approach may also allow international recommendations to be updated. We also considered a crossover design, but a parallel design was finally chosen, given that the crossover design has several limitations. ${ }^{35}$ The crossover design is suitable for patients in a stable condition, but this is not the situation for patients with cancer with NP refractory to opioids and gabapentinoids. We also believe that the treatment might have carryover effects and alter the response to subsequent treatments and that patients may not be in a comparable condition at the start of each treatment period in a crossover trial.

Several issues related to the content of the trial require discussion. There are three major concerns: (i) the heterogeneity of causes of NP, (ii) the choice of the primary endpoint and (iii) the role of depression. To address the heterogeneous causes of NP, we excluded patients with CIPN, but the trial might still be criticised due to combination of peripheral and central NP in one study. Narrower criteria are theoretically possible, but accrual of patients who meet these criteria is likely to be difficult. We thus decided to include both peripheral and central NP in the study, and subgroup analyses will be performed. Second, the primary endpoint is the difference in average pain intensity score at T3 (day 10) between two groups. Although we had acknowledged that some authors recommend worst pain intensity in the last 24 hours as primary endpoints because it satisfies most key recommendations in the draft guidance by the Food and Drug Administration, ${ }^{36}$ average pain intensity is adopted by many clinical trials about $\mathrm{NP},{ }^{37}$ including only one placebo-controlled randomised controlled trial (RCT) in patients with cancer with NP. ${ }^{14}$ Furthermore, to evaluate chronic pain, especially taking into account the nature of NP in this setting, we concluded that it is better to use the 'average pain intensity in the last 24 hours' as the primary endpoint after discussion among the members of the steering committee. Finally, since depression affects the assessment of pain, we excluded patients with depression from the study population for accurate evaluation of the pharmacological effects of duloxetine on pain. Therefore, the planned placebo-controlled, double-blind multicentre RCT will be the first to evaluate the efficacy of pharmacological treatment on well-defined NP in patients with cancer.

\section{Trial status}

The enrolment started in July 2015. At the time of manuscript submission (June 2017), two-thirds of patients have participated. We thus expect to complete the recruitment by December 2017.

\section{Confidentially}

Data will be retained in accordance with the Japanese ethical guidelines for clinical research. Participants will be allocated a unique identification (ID) number at entry. The master list linking participant personal information 
and ID number will be maintained in a separate locked cabinet and password-protected hard drive at each institution. Data will be analysed by ID number only. Records will be retained for 5 years after study completion and then destroyed by the data centre.

\section{Dissemination}

The results of this study will be submitted for publication in international peer-reviewed journals and the key findings presented at conferences. Participants will be informed of the results of the trial by the investigators. Authorship will be ascribed in accordance with the International Committee of Medical Journal Editors guidance.

\section{Author affiliations}

${ }^{1}$ Palliative Care Center, Cancer Center, Kindai University Hospital, Osaka, Japan ${ }^{2}$ Department of Psychosomatic Medicine, Kindai University Faculty of Medicine, Osaka, Japan

${ }^{3}$ Department of Palliative Medicine, National Cancer Center Hospital, Tokyo, Japan ${ }^{4}$ Department of Palliative Medicine, Institute of Medical Science, The University of Tokyo, Tokyo, Japan

${ }^{5}$ Department of Practical Pharmacy, Tokyo University of Pharmacy and Life Sciences, Tokyo, Japan

${ }^{6}$ Department of Palliative Medicine, Kobe University Graduate School of Medicine, Kobe, Japan

${ }^{7}$ Palliative and Supportive Care Division, Seirei Mikatahara General Hospital, Hamamatsu, Japan

${ }^{8}$ Department of Psychosomatic Internal Medicine, National Hospital Organization Kinki-Chuo Chest Medical Center, Sakai, Japan

${ }^{9}$ Department of Clinical Trial Data Management, Graduate School of Medicine, The University of Tokyo, Tokyo, Japan

${ }^{10}$ Japanese Organization for Research and Treatment of Cancer (JORTC), NPO,

Tokyo, Japan

${ }^{11}$ Division of Biostatistics, Tohoku University Graduate School of Medicine, Sendai, Japan

Acknowledgements We are grateful for support from the Japan Agency for Medical Research and Development, Grant-in-Aid for Scientific Research and the Sasakawa Memorial Health Foundation. The authors thank in advance all the patients, investigators and institutions involved in this study.

Contributors $\mathrm{HM}, \mathrm{HI}, \mathrm{SI}, \mathrm{AK}, \mathrm{YK}, \mathrm{TM}, \mathrm{YM}, \mathrm{TM}$ and KA participated in the design of the study. TY and TK designed the statistical analysis plan. All authors contributed to writing and revising the manuscript critically, and all gave their final approval of the version to be published.

Funding This work was supported by the following grants: Sasakawa Memorial Health Foundation Research Grant (Grant No. 2013-A003), 2014-2016 Grant-in-Aid for Scientific Research (Grant-in-Aid for Young Scientists B; Grant No. 26860486), 2014 Health Labour Sciences Research Grant (Grant for Innovative Clinical Cancer Research: Multicenter Clinical Research Concerning the Development of Palliative Medicine for Severe Symptoms in Cancer Patients) Research Team (H26-Innovative Cancer-General-056) and a 2015-2017 Japan Agency for Medical Research and Development award (Innovative Clinical Cancer Research). The entire research organisation has no conflicts of interest to declare.

Competing interests None declared.

\section{Patient consent Obtained.}

Ethics approval The protocol was approved by the institutional review board at each study site. Informed consent for participation in the trial will be obtained from all patients.

Provenance and peer review Not commissioned; externally peer reviewed.

Open Access This is an Open Access article distributed in accordance with the Creative Commons Attribution Non Commercial (CC BY-NC 4.0) license, which permits others to distribute, remix, adapt, build upon this work non-commercially, and license their derivative works on different terms, provided the original work is properly cited and the use is non-commercial. See: http://creativecommons.org/ licenses/by-nc/4.0/
Author note Japanese Organization for Research and Treatment of Cancer (JORTC) Data Center and JORTC Independent Data Monitoring Committee have access to the final trial dataset. There is no contractual agreement regarding investigators' access restrictions on dataset.

(C) Article author(s) (or their employer(s) unless otherwise stated in the text of the article) 2017. All rights reserved. No commercial use is permitted unless otherwise expressly granted.

\section{REFERENCES}

1. Portenoy RK, Miransky J, Thaler HT, et al. Pain in ambulatory patients with lung or colon cancer. Prevalence, characteristics, and effect. Cancer 1992;70:1616-24.

2. García de Paredes ML, del Moral González F, Martínez del Prado P, et al. First evidence of oncologic neuropathic pain prevalence after screening 8615 cancer patients. Results of the On study. Ann Oncol 2011;22:924-30.

3. Fainsinger RL, Fairchild A, Nekolaichuk C, et al. Is pain intensity a predictor of the complexity of cancer pain management? J Clin Oncol 2009;27:585-90.

4. Bennett MI, Rayment C, Hjermstad M, et al. Prevalence and aetiology of neuropathic pain in cancer patients: a systematic review. Pain 2012;153:359-65.

5. Caraceni A, Hanks G, Kaasa S, et al. Use of opioid analgesics in the treatment of cancer pain: evidence-based recommendations from the EAPC. Lancet Oncol 2012;13:e58-e68.

6. O'Connor AB, Dworkin RH. Treatment of neuropathic pain: an overview of recent guidelines. Am J Med 2009;122:S22-S32.

7. Saarto T, Wiffen PJ. Antidepressants for neuropathic pain. Cochrane Database Syst Rev 2007;17:CD005454.

8. The National Comprehensive Cancer network. http://www. oralcancerfoundation.org/treatment/pdf/pain.pdf

9. Dworkin $\mathrm{RH}, \mathrm{O}^{\prime}$ Connor AB, Backonja M, et al. Pharmacologic management of neuropathic pain: evidence-based recommendations. Pain 2007:132:237-51.

10. Tan T, Barry P, Reken S, et al. Pharmacological management of neuropathic pain in non-specialist settings: summary of NICE guidance. BMJ 2010;340:c1079.

11. Attal N, Cruccu G, Baron R, et al. EFNS guidelines on the pharmacological treatment of neuropathic pain: 2010 revision. Eur $J$ Neurol 2010;17:1113-e88.

12. Finnerup NB, Attal N, Haroutounian S, et al. Pharmacotherapy for neuropathic pain in adults: a systematic review and meta-analysis. Lancet Neurol 2015;14:162-73.

13. Perry G, Andrew N, Scott M, et al. Nonopioid pharmacotherapy. Perry G, ed. The diagnosis and treatment of breakthrough pain. New York: Oxford University Press, 2008:86-90.

14. Caraceni A, Zecca E, Bonezzi C, et al. Gabapentin for neuropathic cancer pain: a randomized controlled trial from the Gabapentin Cancer Pain Study Group. J Clin Oncol 2004;22:2909-17.

15. Bouhassira D, Wilhelm S, Schacht A, et al. Neuropathic pain phenotyping as a predictor of treatment response in painful diabetic neuropathy: data from the randomized, double-blind, COMBO-DN study. Pain 2014;155:2171-9.

16. Smith EM, Pang $\mathrm{H}$, Cirrincione $\mathrm{C}$, et al. Effect of duloxetine on pain, function, and quality of life among patients with chemotherapyinduced painful peripheral neuropathy: a randomized clinical trial. JAMA 2013;309:1359-67.

17. Matsuoka H, Makimura $\mathrm{C}$, Koyama A, et al. Pilot study of duloxetine for cancer patients with neuropathic pain non-responsive to pregabalin. Anticancer Res 2012;32:1805-10.

18. Schukro RP, Oehmke MJ, Geroldinger A, et al. Efficacy of duloxetine in chronic low back pain with a neuropathic component: a randomized, double-blind, placebo-controlled crossover trial. Anesthesiology 2016;124:150-8.

19. Tesfaye S, Wilhelm S, Lledo A, et al. Duloxetine and pregabalin: high-dose monotherapy or their combination? The "COMBO-DN study" - a multinational, randomized, double-blind, parallel-group study in patients with diabetic peripheral neuropathic pain. Pain 2013;154:2616-25.

20. Pickering G, Marcoux M, Chapiro S, et al. An algorithm for neuropathic pain management in older people. Drugs Aging 2016;33:575-83.

21. Treede RD, Jensen TS, Campbell JN, et al. Neuropathic pain: redefinition and a grading system for clinical and research purposes. Neurology 2008;70:1630-5.

22. Atkinson TM, Mendoza TR, Sit L, et al. The brief pain inventory and its "pain at its worst in the last 24 hours" item: clinical trial endpoint considerations. Pain Med 2010;11:337-46. 
23. Rao RD, Michalak JC, Sloan JA, et al. Efficacy of gabapentin in the management of chemotherapy-induced peripheral neuropathy: a phase 3 randomized, double-blind, placebo-controlled, crossover trial (NOOC3). Cancer 2007:110:2110-8.

24. Caraceni A, Cherny N, Fainsinger R, et al. Pain measurement tools and methods in clinical research in palliative care: recommendations of an expert working group of the European Association of Palliative Care. J Pain Symptom Manage 2002;23:239-55.

25. Maruo T, Nakae A, Maeda L, et al. Validity, reliability, and assessment sensitivity of the Japanese version of the short-form McGill pain questionnaire 2 in Japanese patients with neuropathic and nonneuropathic pain. Pain Med 2014;15:1930-7.

26. Miyazaki K, Suzukamo Y, Shimozuma K, et al. Verification of the psychometric properties of the Japanese version of the European Organization for Research and Treatment of Cancer Quality of Life Questionnaire Core 15 palliative (EORTCQLQ-C15-PAL). Qual Life Res 2012;21:335-40.

27. Zigmond AS, Snaith RP. The hospital anxiety and depression scale. Acta Psychiatr Scand 1983;67:361-70.

28. Kugaya A, Akechi T, Okuyama T, et al. Screening for psychological distress in Japanese cancer patients. Jpn J Clin Oncol 1998;28:333-8.

29. Utne I, Miaskowski C, Bjordal K, et al. Differences in the use of pain coping strategies between oncology inpatients with mild vs. moderate to severe pain. J Pain Symptom Manage 2009;38:717-26.
30. Matsuoka H, Sakano Y. Assessment of cognitive aspect of pain: development, reliability, and validation of Japanese version of pain catastrophizing scale. Jpn J Psychosoma Med 2007;47:95-102

31. Oken MM, Creech RH, Tormey DC, et al. Toxicity and response criteria of the Eastern Cooperative Oncology Group. Am J Clin Oncol 1982;5:649-56.

32. Toth C. Substitution of gabapentin therapy with pregabalin therapy in neuropathic pain due to peripheral neuropathy. Pain Med 2010;11:456-65.

33. Quilici S, Chancellor J, Löthgren M, et al. Meta-analysis of duloxetine vs. pregabalin and gabapentin in the treatment of diabetic peripheral neuropathic pain. BMC Neurol 2009;9:6

34. Hui D, Shamieh O, Paiva CE, et al. Minimal clinically important differences in the Edmonton Symptom Assessment Scale in cancer patients: a prospective, multicenter study. Cancer 2015;121:3027-35.

35. Senn SS. Cross-over trials in clinical research. Chichester: John Wiley \& Sons, 2002.

36. Atkinson TM, Mendoza TR, Sit L, et al. The brief pain inventory and its "pain at its worst in the last 24 hours" item: clinical trial endpoint considerations. Pain Med 2010;11:337-46.

37. McNicol ED, Midbari A, Eisenberg E. Opioids for neuropathic pain. Cochrane Database Syst Rev 2013;29:8 CD006146. 